\title{
IAMJ
}

INTERNATIONAL

AYURVEDIC

MEDICAL JOURNAL

\section{CLINICAL EVALUATION OF DASAMOOLADI GHRITA VASTI, UPANAHA AND ORAL POLYHERBOMINERAL COMPOUND IN THE MANAGEMENT OF OSTEOARTHRITIS OF KNEE JOINTS (JANU SANDHIGATA VATA)}

\author{
Rima Bhattacharyya ${ }^{1}$, Bishnu Prasad Sharma ${ }^{2}$ \\ PG Scholar, Dept. of Kayachikitsa, Govt. Ayurvedic College \& Hospital, Guwahati-14, Assam, India \\ Guide, Ex-HOD, Dept. Of Kayachikitsa, Govt. Ayurvedic College \& Hospital, Guwahati-14, Assam, India
}

Corresponding Author: bhattacharyyadrrima@gmail.com

https://doi.org/10.46607/iamj05p5042021

(Published online: May 2021)

Open Access

(C) International Ayurvedic Medical Journal, India 2021

Article Received: 31/05/2021 - Peer Reviewed: 31/05/2021 - Accepted for Publication: 31/05/2021

\section{Check for updates}

\section{ABSTRACT}

Sandhigata Vata is the most common form of musculoskeletal disorder. It is a degenerative disease, mostly affects female and the most common age group is in between 35-65 years. Osteoarthritis hampers day to day activities and leads to deformity. Hence, Ayurveda improves the quality of life of osteoarthritis patients through internal and external therapies. In this particular study, Janu Sandhigata Vata patients are treating with Dasamooladi Matra Vasti, Upanaha and oral polyherbomineral compound for a duration of 2 months, in dept. Of Kayachikitsa, Govt. Ayurvedic College and Hospital, Guwahati. The aim of this trial is to establish Ayurvedic Principle of treatment along with various drug options for both internal and external measures to manage Sandhigata Vata. However, further research studies are needed to fulfil the aims and objectives.

Keywords: Janu sandhigata Vata, osteoarthritis, Dasamooladi Matra Vasti, Upanaha, oral polyherbomineral compound, clinical trial. 


\section{INTRODUCTION}

Ayurveda is the Science of life. Health is defined as the state of physical, mental, social and spiritual wellbeing in which disease and infirmity are absent. Among, Tridosha, Vata is responsible for all activities and to create most of the diseases of old age. According to Ayurveda, all Dhatu being undergo degeneration in $4^{\text {th }}$ decade of life. Sandhigata Vata is Vata dominant disease $^{1}$. In the pathogenesis, joints are afflicted by Vata. Sandhi (joints) are one of the types of Marma ${ }^{2}$ and form a part of Madhyama roga marga ${ }^{3}$, makes the disease Kricchasadhya $a^{4}$ or Kastasadhya (difficult to cure).

Osteoarthritis (OA) is a disorder characterized by progressive joint failure in which all structures of the joint have undergone pathologic change ${ }^{5}$. There are numerous pathways that lead to OA, but the initial step is often joint injury in the setting of failure of protective mechanisms.

Aim: To evaluate efficacy of Dasamooladi ghrita vasti, Upanaha and oral Polyherbomineral compound consists of Chandraprabha vati, Prabal bhasma and Amlaki Rasayan.

\section{Objective:}

1. Clinically evaluate the effect of Dasamooladi ghrita vasti, Upanaha and oral polyherbomineral compound in the management of Osteoarthritis of knee joints (Janu sandhigata vata).

2. To decrease the morbidity of the disease and improve quality of life.

\section{Materials and Methods}

1. Sample size: 35

2. Source of data: Total 35 numbers of patients in both OPD and IPD basis, having age in between 25-80 years, irrespective of sex, occupation, religion, in the Dept. Of Kayachikitsa, Govt. Ayurvedic College and Hospital, are selected for clinical trial.

\section{Method of collection of Data:}

Patient fulfilling the inclusion criteria are selected for study. Before starting the treatment, detailed clinical history are taken in the clinical research proforma based on Ayurvedic and modern parameters and the written consent are taken from patients. The study has conducted as open labeled interventional clinical trial after received written consent from The Institutional Ethical Committee.

\section{Inclusion criteria}

1. Patient presenting Classical signs and symptoms of Sandhigatavata ${ }^{6}$ like

$>$ Sandhishoola (pain in joints)

$>$ Sandhishotha (swelling in joints)

$>$ Sandhigraha (stiffness in joints)

> Akunchana Prasarana Vedana (pain during flexion and extension of joints)

$>$ Sparsha Asahyata (tenderness)

$>$ Atopa (Crepitus)

2. Patients fulfilling the modern criteria of diagnosis of OA like dull pain, swelling, pain increases during walking, pain during flexion and extension of joints, stiffness of joints ${ }^{7}$ etc.

3. Patients having two radiological cardinal features like osteophytes and narrowing of space ${ }^{8}$.

4. Patients between age group of 25-80 years.

5. Patients fit for Vasti karma ${ }^{9}$

6. Patients without any previous anatomical deformity.

7. Patients are selected irrespective of sex, religion and occupation.

\section{Exclusion criteria}

1. Patients below 25 years and above 80 years of age.

2. Patients suffering from diseases like carcinoma, psoriatic arthritis, reactive arthritis, infective arthritis, gouty arthritis, tuberculosis, SLE, syphilis, HIV, any cardiac disorders, osteomyelitis, orthopaedic deformity etc.

3. Pregnant lady.

4. Patients are unfit for Vasti procedure.

\section{Diagnostic criteria:}

Diagnosis was made on classical sign and symptom of Janu SandhigataVata6 and Knee Osteoarthritis.

Janu Sandhigata Vata:

* Vata Purna Dhriti Sparsha -on palpation appears as leather bag inflated with air

* Sandhi Shotha- Swelling

* Vedana during Prasarana and Akunchana- Pain during movement of joint

* Shoola - Pain 
Atopa- Crepitus in joint

* Radiological findings such as narrowing of joint space, osteophytes formation at the joint margin, subchondral bone sclerosis, synovitis etc.

\section{Osteoarthritis:}

1. Use related pain affecting one or few joints (rest or noctural pain are less common).

2. Stiffness after rest or in morning may occur but is usually brief $<30$ minutes.

3. Loss of joint movement or functional limitation.

4. Joint instability.

5. Joint deformity.

6. Joint crepitation.

\section{Assessment criteria}

Assessment of the condition has done on a detailed proforma by adopting standard method of scoring of both subjective and objective parameters. These are statistically analysed by using proper statistical method.

\section{Subjective parameters:}

1. Crepitation.

2. Stiffness.

3. Pain during walking, flexion or extension.

4. Swelling.

\section{Objective parameters:}

1. X ray of knee joints, bilateral weight bearing 45-degree flexed position, Lateral, Patella-femoral and Postero-anterior views ${ }^{10}\left(\mathrm{IKDC}^{\mathbf{2 6}}\right)$

\section{Observation and Result}

Total 35 patients were enrolled for the present study. The result of Therapeutic profiles are

Table 1: Effect of therapy on Sandhi shoola (joint pain)

\begin{tabular}{|l|l|l|l|l|l|l|l|l|l|l|}
\hline $\mathbf{N}$ & Mean & \multicolumn{3}{|c|}{ SD } & SE & t value & SE of difference & P value & Remarks \\
\hline 35 & BT & AT & BT & AT & BT & AT & 11.3165 & 0.111 & P< & Significant \\
& 2.63 & 1.37 & 0.49 & 0.49 & 0.08 & 0.08 & & & 0.0001 & \\
\hline
\end{tabular}

\section{WOMAC criteria ${ }^{11}$}

\section{Intervention and Posology: ${ }^{21}$}

1. Chandraprabha vati ${ }^{12}: 500 \mathrm{mg}$ twice a day for 2 months ${ }^{17}$

2. Praval bhasma ${ }^{13}: 250 \mathrm{mg}$ twice a day for 2 months $^{18}$ 3. Amlaki Rasayana ${ }^{14}: 5$ gm twice a day for 2 months 19

Anupana: Warm water / honey ${ }^{20}$

4. Upanaha ${ }^{15}$ with Karanja $^{27}$ (Pongamia pinnata Linn) and Nirgundi (Vitex negundo Linn) has been given on both OPD and IPD basis for 7 days continuously, if needed, this can be repeated after 2 weeks for 2 months ${ }^{22}$

5. Dasamooladi ghrita ${ }^{16}$ matra vasti has been given continuously for 9 days on both OPD and IPD basis, if necessary, Matra vasti can be repeated after 2 weeks for 2 months ${ }^{2324}$.

Dose of Matra vasti: $50 \mathrm{ml}^{25}$

Duration of the study: 2 months

Follow up interval: 2 weeks or if necessary.

Data Analysis: All the data collecting from case history record are placed, analyzed using appropriate statistical tools such as Arithmetic mean, percentage, standard deviation, Paired $\mathrm{t}-$ test and calculate $\mathrm{p}$ (probability) value.

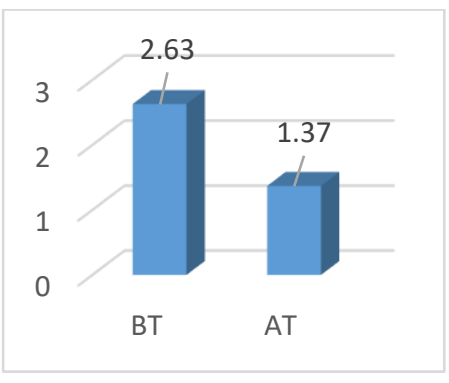

Graph 1: 
Table 2: Effect on therapy on Vata purna driti sparsha (feeling of bag filled with air on touch)

\begin{tabular}{|l|l|l|l|l|l|l|l|l|}
\hline $\mathbf{N}$ & Mean & & SD & SE & t value & P value & Remarks \\
\hline 35 & BT & AT & BT & AT & 0.126 & 7.2851 & P 00.0001 & $\begin{array}{l}\text { Highly } \\
\text { cant }\end{array}$ \\
\hline & 1.14 & 0.23 & 0.88 & 0.23 & & & & Signifi- \\
\hline
\end{tabular}

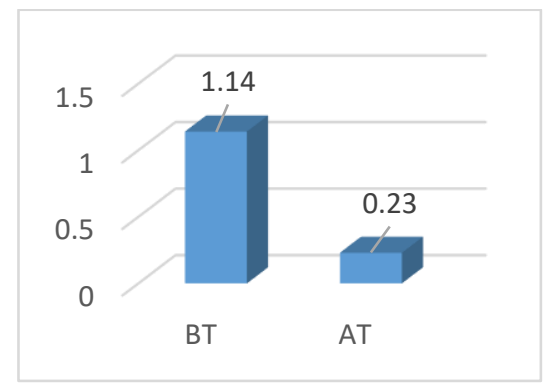

Graph 2:

Table 3: Effect on therapy on Prasarana akunchana vedana (pain on flexion and extension).

\begin{tabular}{|l|l|l|l|l|l|l|l|l|}
\hline $\mathbf{N}$ & Mean & \multicolumn{3}{|l|}{ SD } & SE & t value & P value & Remarks \\
\hline 35 & BT & AT & BT & AT & 0.150 & 9.9107 & P $<0.0001$ & $\begin{array}{l}\text { Highly } \\
\text { cant }\end{array}$ \\
\cline { 2 - 7 }
\end{tabular}

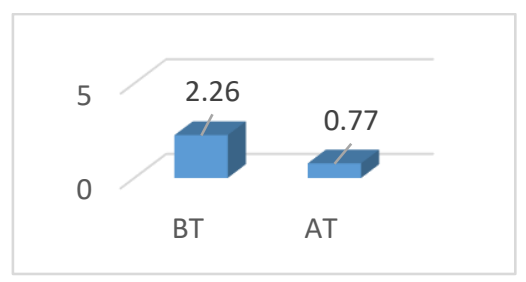

Graph 3:

Table 4: Effect on therapy on Sandhi sotha (swelling):

\begin{tabular}{|c|c|c|c|c|c|c|c|c|}
\hline \multirow{3}{*}{35} & \multicolumn{2}{|c|}{ Mean } & \multicolumn{2}{|l|}{ SD } & SE & t value & $P$ value & Remarks \\
\hline & BT & AT & BT & AT & \multirow[t]{2}{*}{0.143} & \multirow[t]{2}{*}{13} & \multirow[t]{2}{*}{$\mathrm{P}<0.0001$} & \multirow{2}{*}{ Highly Signifi- } \\
\hline & 2.74 & 0.89 & 0.44 & 0.68 & & & & \\
\hline
\end{tabular}

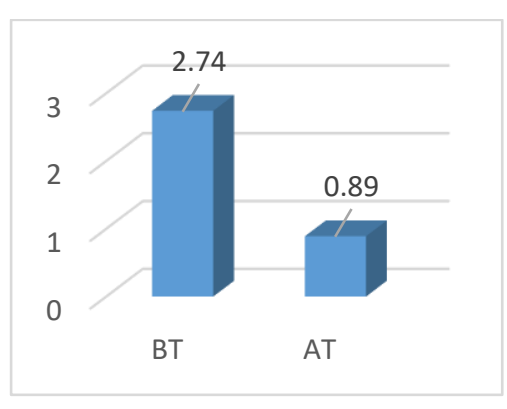

Graph 4: 
Table 5: Effect on therapy on WOMAC scoring.

\begin{tabular}{|l|l|l|l|l|l|l|l|l|l|l|}
\hline $\mathbf{N}$ & Mean & & SD & \multicolumn{3}{|c|}{ SE } & t value & SE of difference & P value & Remarks \\
\hline 35 & BT & AT & BT & AT & BT & AT & 13.0695 & 0.833 & P $<0.0001$ & Significant \\
\cline { 2 - 8 } & 48.14 & 37.26 & 2.87 & 5.68 & 0.49 & 0.96 & & & & \\
\hline
\end{tabular}

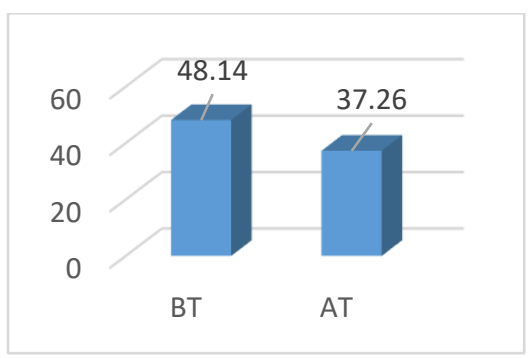

Graph 5:

\section{DISCUSSION}

Sandhigata vata specially occurs among elderly people, in which Dhatukshaya (degeneration) takes place which leads to Vata kopa. Hence, in this study, the main treatment procedure is considered as Matra vasti. Dasamooladi ghrita decreases Sotha, Vedana and stiffness of joints, as it is Vata kapha hara in nature. Upanaha acts as both Bandhana ${ }^{21}$ and Swedana procedure. Karanja $^{27}$ and Nirgundi ${ }^{28}$ are having Vatahara properties, hence they are used in most Vatavyadhis. Percutaneous absorption ${ }^{29}$ of drugs from topical formulation involves the permeation through skin to reach target tissue.

Chandraprabha vati $^{30}$ is Sarvarogahara, Vrishya, Tridosahara and Rasayana. Prabal Bhasma ${ }^{31}$ is coral calyx, it acts on Kshaya Janya Vyadhi (degenerative disease). Prabal bhasma is alkaline in nature and contains calcium as $\mathrm{Ca} 2+$, which is the most compatible form of calcium in body. Amlaki Rasayana ${ }^{32}$ is Vayahsthapana (anti-aging) drug, Tridosahara and Sothahara. It is found significantly to increase grip power of hands and reduced walking time in a research activity.

The result of therapeutic trial showed that- the effect of oral polyherbomineral compound, Upanaha \& Matra vasti with Dasamooladi ghrita is effective. In all criteria $\mathrm{p}$ values are less than 0.0001 and shows highly significant.

\section{CONCLUSION}

Osteoarthritis is more common form of arthritis, affecting millions of people around world. In modern, NSAIDs, Opoids and bone matrix supplements are used for long period, but do not show satisfactory improvement. Oral Ayurvedic Polyherbomineral formulation and Panchkarma procedures are much more convenient to reduce the symptoms and help to repair the joints. However, beyond the present clinical trial, further mass study is required to establish the research drug and procedure, upgrading Principle of treatment of Osteoarthritis.

\section{REFERENCES}

1. Prof Rajeshwardutta Sastri Edited Charaka Samhita of Agnivesh Part 2 Chikitsa sthana $28^{\text {th }}$ chapter Vatavyadhi chikitsa sloka 37 Choukhambha bharati academy Reprinted 2012-page no. 783

2. Ambikadutta Sastri Edited Sushruta Samhita Part 1Sharir sthana chapter 6 Pratyekmarmanirdesha sloka 25 Choukhambha publication Reprinted 2015 ISBN= 978-81-89798-19-2-page no. 72

3. Prof Rajeshwardutta Sastri Edited Charaka Samhita of Agnivesh Part 2 Sutra sthana $11^{\text {th }}$ chapter Trieshaniya sloka 48 Choukhambha bharati academy Reprinted 2012 page no 177

4. Acharya Vidyadhar Shukla Edited Charaka Samhita of Agnivesh Part 1 Sutra sthana $10^{\text {th }}$ chapter Mahachatuspadiya sloka 14-16 Choukhambha bharati academy Reprinted 2011-page no. 177

5. Harrison's principles of internal medicine volume 2 part 11 section 3 chapter 364 David T. Felson Tuhina Neogi Cenveo publisher $20^{\text {th }}$ Edition $I S B N=978-1-259$ 64401-6 page no. 2624. 
6. S.N. Tripathi Edited ShriMadhavakara Madhava nidana chapter 22 Vatavyadhinidana sloka 21 Choukhambha Sanskrit pratisthan Reprinred 2012 ISBN= 978-817084-388-5-page no. 170. Prof Rajeshwardutta Sastri Edited Charaka Samhita of Agnivesh Part 2 Chikitsa sthana $28^{\text {th }}$ chapter Vatavyadhi chikitsa sloka 37 Choukhambha bharati academy Reprinted 2012-page no. 783

7. Harrison's principles of internal medicine volume 2-part 11 section 3-chapter 364 David T. Felson Tuhina Neogi Cenveo publisher ISBN= 978-1-259-64401-6-page no. 2628. Current Medical Diagnosis and Treatment 2017 Chapter 41 Sports medicine and outpatient Orthopaedics Cenveo Publisher $56^{\text {th }}$ Edition $I S B N=978-1-25-$ 958511-1-page no. 1713.

8. Current Medical Diagnosis and Treatment 2017 Chapter 41 Sports medicine and outpatient Orthopaedics Cenveo Publisher 56 ${ }^{\text {th }}$ Edition ISBN= 978-1-25-958511-1-page no. 1714. Robbins and Cotran Pathologic Basis of Disease Chapter 26 Bones, joints and soft tissue tumours Elsevier $8^{\text {th }}$ Edition ISBN= 978-81-312-2491-5-page no. $1236,1237$.

9. Prof Rajeshwardutta Sastri Edited Charaka Samhita of Agnivesh Part 2 Siddhi sthana $2^{\text {nd }}$ chapter Panchkarmiya sloka 19 Choukhambha bharati Academy Reprinted 2012-page no. 984

10. Current Medical Diagnosis and Treatment 2017 Chapter 41 Sports medicine and outpatient Orthopaedics Cenveo Publisher $56^{\text {th }}$ Edition ISBN= 978-1-25-958511-1-page no. 1714.

11. Western Ontario and McMaster Universities Osteoarthritis Index (WOMAC) 1982 https://rozinaandsmithpt.com

12. Rasatantrasara and Siddhaprayoga samgraha Part 1Krishna Gopal Ayurveda Bhavan 26 ${ }^{\text {th }}$ Edition 2016page no. 311,312 Prof Ambikadutta Sastri Edited Bhaisajya Ratnavali of Shri Govindadas chapter 37 Pramehachikitsa sloka 102-110 Choukhambha publisher $18^{\text {th }}$ Edition ISBN= 978-93-86735-39-3-page no. 730

13. Rasatantrasara and Siddhaprayoga samgraha Part 1 Krishna Gopal Ayurveda Bhavan $26^{\text {th }}$ Edition 2016page no. 93-98

14. Rasatantrasara and Siddhaprayoga samgraha Part 2 Krishna Gopal Ayurveda Bhavan 26th Edition 2016page no. 286. Prof Ambikadutta Sastri Edited Bhaisajya Ratnavali of Shri Govindadas chapter 73 Rasayanaprakaranam sloka 25 Choukhambha publisher $18^{\text {th }}$ Edition $I S B N=978-93-86735-39-3-$ page no. 1104

15. Indradeva Tripathi Edited Yogratnakar Vatavyadhi nidanam sloka 363 Choukhambha Krisnadas academy $5^{\text {th }}$ Edition 2019 ISBN= 978-81-218-0217-2-page no. 434

16. Prof Rajeshwardutta Sastri Edited Charaka Samhita of Agnivesh Part 2 Chikitsa sthana $28^{\text {th }}$ chapter Vatavyadhi chikitsa sloka 121Choukhambha bharati academy Reprinted 2012-page no. 799. Shri Jagadiswarprasad
Tripathi Chakradutta $22^{\text {nd }}$ chapter Vatavyadhi chikitsa sloka 91 Choukhambha Sanskrit series $7^{\text {th }}$ Edition ISBN $=978-81-7080-246-6$-page no. 193. Prof Hariharprasad Tripathi Edited Vangasen Samhita chapter 60 Vatarogaadhikar sloka 234 Choukhambha Krishnadas Academy $2^{\text {nd }}$ edition 2016 ISBN= 978-81-7080-301-0page no. 680 .

17. Rasatantrasara and Siddhaprayoga samgraha Part 1 Krishna Gopal Ayurveda Bhawan $201626^{\text {th }}$ Edition page no. 311,312. Shri Sailaja Srivastava Edited Sharangadhar Samhita Madhyama khanda $7^{\text {th }}$ Chapter Vatakakalpanam sloka 5 Choukhambha orientalia ISBN= 978-81-7637-005-9 Reprinted 2017-page no. 107.

18. Rasatantrasara and Siddhaprayoga samgraha Part 1 Krishna Gopal Ayurveda bhawan $201626^{\text {th }}$ Edition page no. 93-98. Shri Vaidyanath Ayurveda Sarasamgraha edition 2018-page no. 139

19. Rasatantrasara and Siddhaprayoga samgraha Part 2 Krishna Gopal Ayurveda bhawan $201626^{\text {th }}$ Edition page no. 286. Shri Sailaja Srivastava Edited Sharangadhar Samhita Madhyama khanda $6^{\text {th }}$ Chapter Curnakalpanam sloka 2-6 Choukhambha orientalia ISBN= 978-817637-005-9 Reprinted 2017-page no. 88,89.

20. Rasatantrasara and Siddhaprayoga samgraha Part 1 Krishna Gopal Ayurveda bhawan $201626^{\text {th }}$ Edition page no. 93-98. Ambikadutta Sastri Edited Sushruta Samhita Part 1 Sutra sthana chapter 46 sloka 425 Chaukhambha publication ISBN= 978-81-89798-19-2 Reprited 2015page no.279,

21. Ambikadutta Sastri Edited Sushruta Samhita Part 1 Chikitsa sthana chapter 4 Vatavyadhichikitsa sloka 8 Chaukhambha publication ISBN= 978-81-89798-19-2 Reprinted 2015-page no.34. Shri Jagadiswarprasad Tripathi Chakradutta $22^{\text {nd }}$ chapter Vatavyadhi chikitsa sloka 9 Choukhambha sanskrit series $7^{\text {th }}$ Edition ISBN= 978-81-7080-246-6-page no. 193.

22. Ambikadutta Sastri Edited Sushruta Samhita Part 1 Chikitsa sthana chapter 32 Swedaavasaraniya sloka 12 Choukhambha publication ISBN= 978-81-89798-19-2 Reprinted 2015 page no. 174.

23. The Ayurvedic Pharmacopoeia of India Part $12^{\text {nd }}$ Edition page no. 225,226, 227, 254

24. Prof Rajeshwardutta Sastri Edited Charaka Samhita of Agnivesh Part 2 Siddhi sthana $3^{\text {rd }}$ chapter Vastisutriya sloka 24-25 Choukhambha bharati academy Reprinted 2012-page no. 994. Atrideva Gupta Edited Astanga Hridaya Sutra sthana Chapter 19 Vastividhi sloka 25,26 Choukhambha prakashan Reprinted 2017 page no. 164

25. Atrideva Gupta Edited Astanga Hridaya Sutra chapter 19 Vastividhi sloka 27, 28, 29 Choukhambha publication Reprinted 2017-page no. 164.

26. International Knee Documentation Committee (IKDC)subjective score system $1993 \mathrm{https}: / / \mathrm{www} . r e-$ searchgate.net/publication/265391129_The_measurement_properties_of_the_IKDC-subjective_knee_form 
27. "Phytochemical and Pharmacological studies on Pongamia pinnata" 2018 ISSN= 2250-1991 Paripex-Indian journal of research www.worldwidejournals.com

28. "Comprehensive study of Nirgundi plant: A survey report" Journal of Innovations in Pharmaceuticals and Biological sciences ISSN = 2349-2759 www.jipbs.com

29. "Transdermal drug delivery system: a review" The Pharma Innovation ISSN= 2277-7695 vol 1 No.4 2012 www.thepharmajournal.com

30. "Pharmaco-Therapeutic profiles of Chandraprabha vati - An Ayurvedic herbomineral formulation" International Journal of Pharmaceutical \& Biological archieves 2012 ISSN= 0976-3333 www.ijpba.info

31. "Qualitative analysis of praval bhasma bynamburi phased spot test" Journal of Pharmacognosy \& Phytochemistry ISSN= 2278-4136 www.phytojournal.com

32. "Antiaging effect of Amlaki Rasayana in healthy elderly subjects" JAHM ISSN= 2321-1563 www.jahm.in

\section{Source of Support: Nil \\ Conflict of Interest: None Declared}

How to cite this URL: Rima Bhattacharyya \& Bishnu Prasad Sharma: Clinical Evaluation Of Dasamooladi Ghrita Vasti, Upanaha And Oral Polyherbomineral Compound In The Management Of Osteoarthritis Of Knee Joints (Janu Sandhigata Vata). International Ayurvedic Medical Journal \{online\} 2021 \{cited May, 2021\} Available from: http://www.iamj.in/posts/images/upload/2902 2908.pdf 